\title{
HUMANISMO, UN ESFUERZO EN LA vida de Carlos GutiérReZ-Noriega
}

\author{
Humanism a Carlos Gutiérrez-Noriega's life endeavor
}

\author{
Salomón Ayala Pío ${ }^{1}$ y Jeff Huarcaya Victoria ${ }^{2}$
}

\begin{abstract}
Resumen
El presente estudio es una aproximación histórica de la vocación humanista del investigador Carlos Gutiérrez-Noriega, considerando su trayectoria sobresaliente en la investigación científica original y el cultivo de las humanidades. Destacó en diversos escenarios del saber; las ciencias básicas en los laboratorios de farmacología, el estudio de las características y problemas propios del hombre andino y el cocaísmo en sus aspectos biológicos, psicológicos, culturales, antropológicos y sociológicos. Se invita a nuestras pasadas y nuevas generaciones a reflexionar sobre la trascendente experiencia del profesor Gutiérrez-Noriega durante su vida y tiempo.
\end{abstract}

Palabras claves: Humanismo, farmacología, historia, medicina peruana.

Abstract

This study is a historical approach of the researcher Carlos Gutiérrez-Noriega's humanistic vocation, considering his outstanding path in the original scientific research and humanities. He prospered in several knowledge fields, the basic sciences in the pharmacology laboratories, the study of the characteristics and problems of the Andean people and cocaism in biological, psychological, cultural, anthropological and social aspects. Our old and new generations are invited to think about the professor Gutiérrez-Noriega's transcendent experience during his life and time.

Key words: humanism, pharmacology, history, Peruvian medicine.

“... los descubrimientos de las ciencias físicas y naturales están transformando el mundo a tal extremo, que puede originarse un serio desequilibrio si los progresos en el control de la

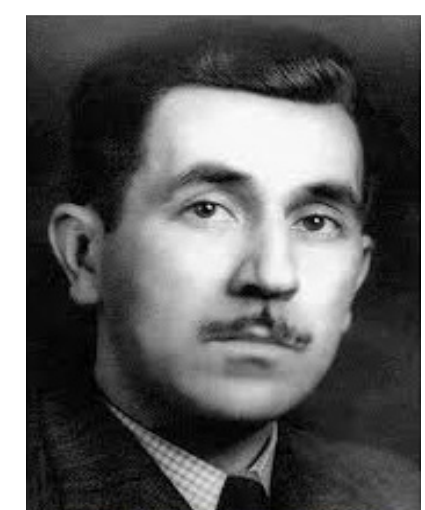

Carlos Gutiérrez-Noriega, (1906-1950)

materia no se compensan con la creación de nuevos valores en la mente."

Carlos Gutiérrez-Noriega (Discurso de Orden de la Presidencia de la Sociedad Peruana de Neuropsiquiatría, 1946)

1 Médico gastroenterólogo. Ex profesor. Facultad de Medicina, Universidad Nacional Mayor de San Marcos y Universidad San Martín de Porres, Lima.

2 Médico psiquiatra. Facultad de Medicina, Universidad San Martín de Porres, Lima. 
para las escuelas alemanas centrado en un estudio de las lenguas y autores "clásicos" (latín y griego). ${ }^{1}$ Esta concepción de humanismo tiene una raigambre pedagógica; se esperaba que, mediante el cultivo de la gramática, la poética, la retórica, la filosofía y la historia se logre dar plenitud a la condición humana. 2 Sin embargo, previo a esta concepción, el movimiento humanista renacentista fue quizá uno de los más influyentes, el cual generó en los siglos XV y XVI los studia humanitatis, redescubriendo a varios autores clásicos griegos y latinos. ${ }^{3}$ Se rompieron las tradiciones escolásticas medievales y se exaltaron las cualidades de la naturaleza humana, lo que reafirmaba su dignidad al buscarle un sentido racional a la vida. La corriente humanista, iniciada en el renacimiento europeo con Petrarca y Alighieri, se enriqueció con los descubrimientos de Galileo, Vesalio, Leonardo da Vinci, entre otros, lo que permitió a las personas liberarse del corsé religioso medieval, se inició también el camino a la ciencia y el método científico que desembocó luego en la corriente del positivismo, que en exceso puede hacer declinar al humanismo. ${ }^{4}$ Erich Fromm habló del grito de ira de quienes creen que las condiciones para una vida digna y humana están desapareciendo. Las experiencias deshumanizantes vividas por los seres humanos tienden a ser los mismos en todas las partes del planeta; de tal manera que el humanismo contemporáneo es una respuesta a dicha deshumanización, con lo que se pretende rescatar la dignidad del ser humano, para lo cual recurre a la formación cultural en el sentido amplio de la palabra con inclusión de las artes, y en particular de la psicología, la antropología, la filosofía, la sociología, la historia, la literatura, pero con poco éxito. ${ }^{4}$ Con respectoalamedicina, noesningún misterioque actualmente nos vemos enfrentados a severas limitaciones en la práctica médica, ya que, por un lado, tenemos avances científico-técnicos

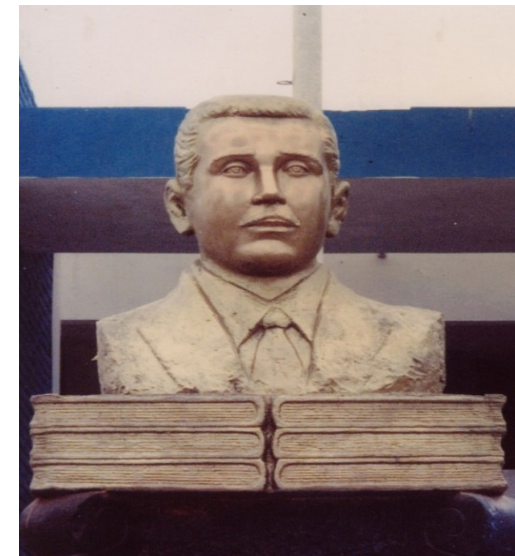

Busto de Carlos Gutiérrez-Noriega en la Gran Unidad Escolar "Carlos Gutiérrez Noriega", Chepén, Trujillo, en la que se resalta el simbolismo de su erudición. (Salomón Ayala Pío, 2010).

que facilitan la investigación y tratamiento de las enfermedades pero, también, nos hemos centrado excesivamente en enfermedades $y$ órganos, llegando en última instancia a un reduccionismo físico del paciente, siendo todo esto factores que favorecen una práctica médica "deshumanizada". ${ }^{5}$ Ya lo señaló Seguin: “... el progreso de la sociedad hacia el cientificismo no se ha cumplido sin sacrificar, en el camino, lo más auténtico de la humanidad y sin conducirnos a convertir al hombre... en una máquina reparable por un técnico". ${ }^{6}$ Es importante resaltar, en el contexto de la práctica médica contemporánea, figuras ejemplares que hayan logrado una visión humanista con la finalidad que viejas y nuevas generaciones puedan reflexionar sobre nuestra historia psicológica descubriendo la autognosis y la ética, así como la fuerza educativa y creativa. Es así que, en el presente artículo, buscamos rescatar la visión humanista del ilustre maestro sanmarquino Carlos Gutiérrez-Noriega, (1906-1950), al revisar algunos de sus aportes académicos en las áreas de psiquiatría y psicología transcultural, literatura, farmacología general y sus estudios sobre el cocaísmo en el Perú. 


\section{Contexto Social, Político y Científico}

Los estudios universitarios de GutiérrezNoriega se dieron en una época de gran conflicto político y universitario en el contexto del gobierno de Benavides (1933-1939), quién reabrió la Universidad Nacional Mayor de San Marcos(UNMSM) en 1935, la cual seencontraba clausurada desde 1932, y expidió un nuevo Estatuto Universitario, de corte autoritario, que limitó la autonomía universitaria, suprimió el cogobierno y el derecho de tacha de los alumnos. Por este motivo Gutiérrez-Noriega cursó el último año de medicina en Chile. En lo cultural, el balance de los años 1930 fue de infertilidad, oscurantismo, fue la época de Sánchez Cerro y de su sucesor Benavides que Pablo Macera los bautizó como la "generación clausurada" .

Después de la Segunda Guerra Mundial, con la amplia amnistía al movimiento aprista peruano dado en el gobierno de Bustamante (1945-1948), se dio la facilidad para que Luis Alberto Sánchez asumiera el rectorado de la UNMSM de 1945 a 1948, y la elección de Sergio Bernales al Decanato de la Facultad de Medicina, constituyendo el máximo apoyo político y universitario para los trabajos de Gutiérrez-Noriega, que se interrumpieron en 1948 con el golpe de estado de Manuel Odria. ${ }^{8}$ Por otra parte, la primera mitad del Siglo XX fue el momento de mayor desarrollo investigativo e intelectual en el colectivo médico peruano, destacando la patología nacional con la enfermedad de Carrión y las enfermedades de la altura. ${ }^{9}$ Hacia el final de esta época, junto con Pedro Weiss, Honorio Delgado, Carlos Monge Medrano, Alberto Hurtado, entre otros, se hizo posible el desarrollo de la Escuela Médica Peruana (1886-1956).

\section{Primeros Trabajos}

La inclinación por el arte y la cultura en Gutiérrez-Noriega apareció desde muy temprano, en su etapa de estudiante y líder del Centro de Estudiantes de Medicina de la UNMSM. Uno de sus primeros trabajos fue el ensayo "Hacia una concepción biológica del arte" publicado en la revista Amauta en 1929, el cual ha sido reproducido en el libro Cultura y Personalidad de la UNMSM en el 2010. En las conclusiones refirió:

"...el arte, más que otros hechos biológicos, se tiene evidente la extraversión dirigida en sentido diferente a la necesidad especifica. En muchos otros fenómenos de esta naturaleza abundan estos residuos más o menos considerables que no siguen las leyes de Darwin ni físicoquímicas, pero ningún otro como los artístico se encuentra dotado de una capacidad tan grande para apartarse de ellas". ${ }^{10}$

Por lo tanto, las obras de arte, no pueden ser reducidas a necesidades ni a fenómenos físicoquímicos. Es probable que Gutiérrez-Noriega llevara esta primera consideración al terreno de lo humano, ya que las personas, al igual que las obras de arte, no pueden ser reducidas a sus elementos formativos. Este pensamiento fue directriz en su vasta obra científica. Entre otros ensayos de inspiración psicológica, presentó "Psicoanálisis de la cultura", en el cual explicó, de manera psicoanalítica, la mentalidad revolucionaria de su época y la del marxismo en particular. ${ }^{11}$ No aceptó la teoría de Adler, para quien el movimiento universitario es resultado de "pobreza e inhabilidad espirituales". ${ }^{11}$ Realizó, además, una reflexión panorámica de las culturas grecolatinas, medieval y moderna; de las influencias del ello, el yo y el superyo sobre las evoluciones de los individuos y sociedades, discrepando con Spengler en la caída de occidente. ${ }^{11}$ 


\section{Profesor Universitario}

Habiendo ingresado a la UNMSM en 1924, su labor docente se inició tempranamente, en la cátedra de fisiología, como ayudante y jefe de trabajos experimentales, notándose desde ese entonces su interés en la fisiología experimental y su habilidad juvenil para el trabajo de laboratorio, siendo aún estudiante de medicina. Luego de graduarse con una tesis de contenido psicoanalítico en 1935, pasó a la cátedra de farmacología donde ascendió hasta profesor principal y jefe de la cátedra interino en 1938. En esta época escribió artículos sobre docencia e investigación, como "Origen e historia de las teorías farmacológicas"12, en la que hizo un breve análisis de la evolución y el logro de la fisiología y farmacología desde la antigüedad. En 1938 publicó "La investigación y la enseñanza de la farmacología en Estados Unidos", ${ }^{13}$ notándose la influencia de la educación estadounidense en la investigación y trabajo en equipo. En 1944 publicó "Posición actual de la farmacología en la medicina y en la biología" ${ }^{14}$ En 1946 plasmó su esfuerzo docente en el primer libro texto de "Farmacología y sus Aplicaciones Terapéuticas" publicado en el Perú para estudiantes y médicos. En 1950 publicó una segunda edición. Campos mencionó: “... llevó su actividad académica con auténtica pasión... una vida dedicada al conocimiento científico y humanistico, por lo que el maestro Carlos GutiérrezNoriega representa, en su connotación más integra y universal, el paradigma del profesor universitario" ${ }^{15}$

\section{Psiquiatría y Psicología Transcultural}

Gutiérrez-Noriega intervino, en el año de su graduación, en diversos proyectos de información en la revista Actualidad Médica Peruana, liderada por Sergio Bernales, en la que se desempeñó como editorialista y años después como director. En una nota editorial de dicha revista señaló algunas preocupaciones en el marco de la actividad de investigación, siendo la principal el excluir la antropología y la sociología de las investigaciones médicas; no ocupándose de la realidad del hombre social, la medicina convierte su estudio en especulación de gabinete, de clínica, de laboratorio. Se ve solo lo cercano, se elimina el gran panorama de la vida colectiva. El centro de preocupación de la revista era el desarrollo de una adecuada aproximación al estudio de las poblaciones indígenas, como el más vasto e importante de los problemas médico-sociales a atender para la "reconstrucción de muchas naciones americanas". ${ }^{16}$

En 1936 presentó el ensayo "El temperamento ciclotímico y el tipo pícnico entre los aborígenes peruanos", en el cual, siguiendo la tipología de Kretschmer, describió los ceramios Mochicas, los cuales mayoritariamente pertenecieron al tipo pícnico, anotó que el temperamento ciclotímico es extravertido, de imaginación realista, mientras que el esquizotímico es autista, introvertido y abstracto. ${ }^{17}$ Este ensayo es complementado con otra publicación: "Biotipología del Perú antiguo", en el cual dio un atisbo del estudio biotipológico y psicológico del indio peruano. ${ }^{18}$

En 1937, a los dos años de su graduación, presentó un ensayo de gran densidad intelectual titulado "Diseño de un estudio psicológico y antropológico de la raza amerindia", en el cual realizó una crítica frontal al medio académico universitario de aquel entonces por el poco interés de realizar una investigación integral del indígena. ${ }^{19}$ Indicó que la hipoafectividad, la introversión, la nostalgia y un predominio del temperamento esquizotímico eran características de la población indígena. ${ }^{19}$ También destacó en esta línea sus estudios sobre la caracterología de otros círculos culturales "Tres tipos culturales de personalidad", de aparición póstuma, en 
que se perfila las personalidades de la cultura occidental, indostánica y sionista, que fue prologado por Honorio Delgado. ${ }^{20}$ En otro estudio titulado "Observaciones biotipológicas y psicopatológicas en los enfermos mentales peruanos con referencia a los factores raciales y geográficos" realizado en 600 pacientes del Hospital Víctor Larco Herrera, utilizó el enfoque de Kretschmer, describiendo la esquizofrenia y la melancolía más frecuentes en el grupo andino y la manías en el costeño. ${ }^{21}$ En 1939, en "El pensamiento mágico en las pinturas del antiguo Perú" realizó una interpretación del arte incaico. ${ }^{22}$

En 1942 fundó, junto con Telémaco Batistini, la Revista de Medicina Experimental del Instituto Nacional de Salud y, en 1947, la Revista de Farmacología y Medicina Experimental de su naciente Instituto. En este período GutiérrezNoriega estuvo inmerso en la influencia de un dualismo evidente y significativo de profundo valor antropo-geográfico. En su artículo "La concepción del mundo y la reacción espiritual congruentes al medio geográfico en el antiguo Perú", señaló quelas formas de cultura y los tipos psicológicos de hombre se elaboraron según el ambiente y para el ambiente, no encontrándose un todo uniforme en sus grandes regiones de costa y sierra. ${ }^{23}$ En esta línea de concepción universal, a finales de su vida, publicó un ensayo de la cultura china en "Sabiduría, humanismo y bondad de Po Chu-i", uno de los más grandes poetas chinos (772-846 AC), de origen humilde, que llegó a alcanzar altos cargos en el gobierno imperial y que le permitió observar la política de ese entonces, siendo su poesía más famosa la de protesta social, aunque muchas son privadas y de meditación. ${ }^{24}$ Todos estos intereses antropológicos fueron corroborados por Guerra-García quien, al visitar la biblioteca del puerto de Pacasmayo, al final de los años noventa, encontró parte de la biblioteca de Gutiérrez-Noriega, en la cual figuraban diversas obras dedicadas al estudio de las religiones, el arte, la filosofía y la ciencia. ${ }^{25}$

En suma, Gutiérrez-Noriega buscó crear una identidad del hombre peruano en base a sus investigaciones. Según Mariátegui, lo fundamental desu legado científico se encuentra en los "diseños" o "bocetos" acerca del hombre peruano. ${ }^{26}$ Estudioso del indigenismo, trató de comprender los más vitales aspectos de la realidad indoamericana. Como señaló Valdivia: "Gutiérrez-Noriega fue un gran peruanista y sus investigaciones llevan la dirección de explorar nuestra realidad humana nacional en su patología psíquica, en sus hábitos, creencias, costumbres y tradiciones". ${ }^{27} \mathrm{Si}$ entendemos al humanismo como un rescate de la "dignidad humana", vemos como Gutiérrez-Noriega, a través de sus estudios psiquiátricos y psicológicos, los cuales tuvieron una gran visión antropológica, buscó rescatar la "dignidad" del hombre indígena, tal cual lo hizo anteriormente Hermilio Valdizán (1885-1929).

\section{El Cervantismo en Gutiérrez-Noriega}

El hecho de que muchos personajes de las novelas de Miguel de Cervantes (1547-1616) padeciesen de aparentes trastornos mentales ha suscitado la curiosidad de muchos estudiosos de la mente humana. ${ }^{28}$ Recordemos que Freud decidió aprender el castellano con la finalidad de poder leer personalmente las obras de Cervantes. Gutiérrez-Noriega no fue ajeno a este interés por la obra cervantina, de la cual fue un admiradoryestudioso. Publicó "La contribución de Miguel de Cervantes a la psiquiatría"29, "Contribución de Cervantes a la psicología y a la psiquiatría"30, "Contribución de Cervantes a la Psicología Médica" 31, "La personalidad y el carácter en la obra de Cervantes"32, "Significado y trascendencia del humorismo en la obra de Cervantes" 33 y "Psicologías anormales en la novela picaresca española del siglo de oro". ${ }^{34}$ 
Por su alta calidad académica, estos ensayos fueron reconocidos a nivel internacional. Vallejo mencionó que, de entre todos los trabajos realizados por eminentes psiquiatras sobre la obra cervantina, los de Gutiérrez-Noriega son los más interesantes. ${ }^{28}$ Para GutiérrezNoriega, Cervantes realizó observaciones psicológicas y psicopatológicas tan originales e importantes que permiten considerarlo como uno de los principales precursores y maestros en la ciencia psiquiátrica. ${ }^{28}$ Según GutiérrezNoriega, lo substancial de la obra de Cervantes radica en la caracterología psicopatológica y psicológica, revelando una clasificación de las personalidades "que no sólo supera a las antiguas doctrinas hipocráticas sino que es la base de la actual caracterología". ${ }^{32}$ Los casos que describió Cervantes no tuvieron como base a los conocimientos teóricos de la psicología antigua, su verdadera contribución viene de las observaciones personales, casi de orden empírico, que él realizó por su propia cuenta en las "casas de orates" o "loquerías". Gutiérrez-Noriega señalo que la fina observación de Cervantes le permitió describir varias alteraciones mentales como el delirio sistematizado, las alteraciones de la percepción, los temperamentos con anticipo a la moderna tipología, así como la concepción mágica y demonológica. ${ }^{29-33}$ Como mencionó Bailón, el cervantismo de Gutiérrez-Noriega se fundamentaba en la antropología, la perspectiva médica, psiquiátrica y humanística. ${ }^{35}$

\section{Estudios En Etnofarmacología, Psiquiatría EXPERIMENTAL Y FARMACOLOGÍA GENERAL}

En 1936 inició sus estudios farmacológicos en animales de experimentación de plantas medicinales peruanas, como la Vallesia dichotoma (Cuncuna). Siguió una serie -que se encuentra mencionada en una revisión previa- y continuó con el estudio de plantas de conocidos efectos alucinógenos en el Perú, como el toe, la Opuntia cylindrica hoy Trichocereus pachanoi San Pedro y la ayahuasca. ${ }^{36}$ Con el San Pedro realizó varios estudios de alteraciones mentales y psicosis experimental.

Profundizó en el estudio del cardiazol, tratando de desentrañar los mecanismos de las convulsiones experimentales a diferentes niveles del sistema nervioso, en numerosas publicaciones de neurofisiología en la escala animal, que condensó en su tesis doctoral de 1940: "Fisiopatología y neurobiología de la epilepsia producida por cardiazol". ${ }^{37}$ También fue objeto de sus estudios otros fármacos como la nicotina, fármacos colinérgicos, vagotonina, yohimbina, morfina, adrenalina, benzedrina, coramina, estricnina, bulbocapnina, analépticos, fenobarbital, etc.; todos los cuales fueron realizados en la cátedra de farmacología de la UNMSM y en el laboratorio de medicina experimental del Instituto Nacional de Salud. ${ }^{36}$

\section{Estudios SOBRe Cocaísmo y Cocainismo}

Gutiérrez-Noriega inició sus estudios sobre la coca en 1937 y luego los retomó en 1944. Condensó sus trabajos en su libro premiado "Estudios sobre la coca y la cocaína en el Perú" publicado en $1947^{38}$, en el cual presentó un estudio integral en los aspectos fisiológicos, farmacológicos, patológicos y sociales de la coca, siendo el documento más completo de la época. Sobre cocaísmo y cocainismo produjo con sus colaboradores, Vicente Zapata Ortiz (principal asistente), Rotondo, Ciuffardi, Chamochumbi, Salomón, Cruz Sánchez, Pons Muzo, Vargas Machuca, Risemberg, y otros de su equipo, un total de 26 trabajos (1944-1952), los cuales sistematizamos en una revisión previa. ${ }^{39}$ Se considera a Gutiérrez-Noriega como el primer investigador del coqueo en el Perú y en el mundo. 
En su artículo "La coca y la alimentación en el Perú" señaló: "El estudio de la habituación de una droga requiere diversos métodos de investigación, constituye un fenómeno social complejo, deben investigarse desde los puntos de vista históricos, sociológicos, antropológicos, estadísticos y biológicos. En el Perú, los factores sociológicos del cocaísmo -la droga que mitiga el hambre y la fatiga- están en relación con aspectos antropogeográficos, culturales, económicos, psicosociales y nutritivos. Según la tesis que se expone en el presente estudio, el cocaísmo, se ha desarrollado a consecuencia de una merma de mala alimentación, la que a su vez fue consecuencia de ciertos acontecimientos históricos". ${ }^{40}$ En una reciente investigación histórica académica, "Gutiérrez-Noriega y el debate en torno al consumo de hojas de coca (1937-1952)", analizamos las evidencias producidas por Gutiérrez-Noriega y su equipo sobre las implicancias sanitarias desfavorables del consumo de coca para el pueblo peruano y su repercusión en la política nacional e internacional. ${ }^{39}$ Estos excelentes trabajos sobre el cocaísmo de los años 1940 y 1950 deben ser apreciados en su contexto y su tiempo, y, como todo conocimiento humano, las investigaciones son parciales y tendenciosas, siendo el mismo Gutiérrez-Noriega quien reconoció que sus trabajos deben interpretarse en el contexto de las condiciones de vida de la época. ${ }^{41}$

\section{RefleXiones FinAles}

En el presente artículo presentamos el avance en el sitial de la ciencia y de las humanidades como una dimensión fundamental de la vida del maestro Gutiérrez-Noriega. Creyó que la mejor evidencia disponible para el progreso humano es producto de las investigaciones a la que se dedicó apasionadamente expresando su vocación humanista peruana, latinoamericana y universal. Bernal mencionó que GutiérrezNoriega manifestó un "especial interés en la persona humana, concretamente el peruano, en su medio y todo lo que podría ser de valor para ayudar a su conocimiento, y contribuyó como pocos a incrementar el acervo de la cultura universal". ${ }^{42}$ Mariátegui mencionó que la obra de Gutiérrez-Noriega parte de una perspectiva antropológica en procura de una perspectiva global, comprensible y ecológica. ${ }^{26}$ Vemos como la obra de Gutiérrez-Noriega se encuentra en consonancia con lo que mencionó el historiador alemán Rüsen, para quien la noción del humanismo debería situarse siempre en su contexto social para que tenga verosimilitud y ocupar el puesto que le corresponde en la vida real, de esta forma, el humanismo no debería concebirse como una empresa exclusivamente circunscrita a los círculos científicos e intelectuales. ${ }^{43}$ Los trabajos de GutiérrezNoriega y sus colaboradores sobre cocaísmo y los efectos nocivos entre nuestros indígenas han recibido reconocimiento internacional. Estudió el hábito del chacchado en todos sus aspectos y en sus consecuencias más importantes, desde los puntos de vista químico, fisiológico, farmacológico, psicológico y médico-social. Los resultados de sus investigaciones figuraron en los capítulos correspondientes de algunos tratados de farmacología de reputación internacional. ${ }^{44}$

Permitiéndonos una digresión sobre la actualidad, respecto a las competencias interculturales en la Facultad de Medicina de la UNMSM, Murillo realizó un planteamiento de las bases conceptuales y metodológicos de dichas competencias. ${ }^{45}$ Como antecedente tenemos a Seguin, quien defendió, en los debates sobre el nuevo plan de estudios en 1964, que era necesaria una formación integral de los alumnos de medicina, por lo que se debía incluir en el plan de estudios contenidos de sociología y antropología a fin de lograr un entendimiento de la enfermedad en el contexto social y cultural de los pacientes. ${ }^{46}$ Por otra parte, Nizama, 
en una extensa publicación actual sobre "Humanismo Médico", señaló lo perjudicial de la deshumanización de la sociedad globalizada y la insensibilidad humana, siendo necesario potenciar la formación humanista durante la carrera de medicina, enfatizando los principios éticos y el perfil del médico humanista. ${ }^{47}$ Gutiérrez-Noriega proyectó con sus trabajos una dimensión nacional con su gran compromiso con la universidad y el país. Falleció joven, a los 44 años -al igual que Valdizán-, quedando truncada su obra. Zapata mencionó que "la muerte lo reclamó cuando se encontraba en plena evolución constructiva y aunque son muy valiosas las contribuciones que ha legado, era de inmenso valor la obra que se esperaba de él en el porvenir". ${ }^{4}$ El maestro fue enterrado en San Pedro de Lloc, ubicado en Pacasmayo, Trujillo, figurando en su lápida: "Sabio fallecido trágicamente en Pisa, Italia, el 26 de octubre de 1950, después de tomar parte en el Congreso Mundial de Psiquiatría, en París, representando a su Patria. Como farmacólogo y psiquiatra hizo valiosas contribuciones a la ciencia, destacando como un auténtico benefactor del indio por luchar contra el cocaísmo". A pedido de Honorio Delgado la Biblioteca Nacional publicó la Bio-bibliografía del Gutiérrez-Noriega. Al poco tiempo de su muerte, Cruz documentó las publicaciones científicas de GutiérrezNoriega en un total 120 artículos y 10 inéditos. ${ }^{49}$ Posteriormente, Valdivia logro documentar hasta 153 artículos..$^{50}$

Finalmente, ¿Qué era el humanismo para Gutiérrez-Noriega? Si bien podríamos dar una respuesta de forma indirecta luego de haber revisado su vasta obra, podemos suponer que la idea de humanismo que tenía era similar a la que expresó respecto a la "Edad de oro del humanismo:

... Lo fundamental para la evolución espiritual del hombre, y que constituye el origen de todas las disciplinas psicológicas, es que tanto los orientales como griegos, proclaman el conocimiento y perfección de sí mismos, y la autarquía de la personalidad como fundamentos del humanismo, es decir, de la conciencia y personalidad del hombre actual...".$^{51}$

Como mencionó Marañón: "el hombre de ciencia, que solo es hombre de ciencia, y que progresa, creerá que su obra es el centro del universo y perderá el contacto generoso con la realidad ajena, podia caer en vanidad y petulancia, para que esto no ocurra, tener el alivio de una vena permanente y fresca de preocupaciones universales, he aqui la mente humanista". ${ }^{52}$ Así vivió Gutiérrez-Noriega, sencillo y modesto, alejado de ornamentales títulos académicos y de exposición mediática, con intereses humanísticos que no fueron exclusivamente teóricos y metafísicos, sino pragmáticos buscando ejercer una influencia en nuestro modo de pensar y entender a los pobladores peruanos.

\section{REFERENCIAS BibliogRÁficAS}

1. Ferrater J. Diccionario de filosofía. 5a ed. Buenos Aires: Editorial Sudamericana; 1965. Humanismo. p. 875-78.

2. Ginzo A. Humanismo filosófico y Renacimiento. Indagación: Revista de historia y arte. 1994; 0: 85-98.

3. Cordua C. El humanismo. Rev chil lit. 2013; 84: 9-17.

4. Díaz A. Qué significa una medicina más humana en tiempos de la Industria Salud-Enfermedad. Arch Med Interna. 2015; 37(2): 83-6.

5. Wagner P, Perales A, Armas R, Codas O, de los Santos R, Elio-Calvo D, et al. Bases y perspectivas latinoamericanas sobre Medicina y Salud Centradas en la Persona. An Fac med. 2015; 76(1): 63-70.

6. Seguin CA. Medicinas tradicional y medicina folklorica. Lima: Banco Central de Reserva del Perú - Fondo Editorial; 1988.

7. Salaverry O, Chiri S. Entrevista a Estuardo Nuñez Hague. Alma Mater.1999; 16: 27-8.

8. Bustios C. La Salud Pública, la Seguridad Social y el Perú Demoliberal (1933-1968). Lima: CONCYTEC; 2005. p. 243.

9. Cueto M. Excelencia científica en la periferia. Actividades científicas e investigación biomédica en el Perú. 18901950. Lima: GRADE-CONCYTEC; 1989.

10. Gutiérrez-Noriega -Noriega C. Hacia una concepción biológica del arte. En Mariátegui J, Guerra-García R. 
Cultura y Personalidad - Escritos conexos. Lima: Fondo Editorial Universidad Nacional Mayor de San Marcos; 2010. p. 25-61.

11. Gutiérrez-Noriega C. Psicoanálisis de la cultura. En Mariátegui J, Guerra-García R. Cultura y Personalidad - Escritos conexos. Lima: Fondo Editorial Universidad Nacional Mayor de San Marcos; 2010. p. 71-80.

12. Gutiérrez-Noriega C. Origen e historia de las teorías farmacológicas. Actual Med Peru.1938; 4(8): 271-85.

13. Gutiérrez-Noriega C. La investigación y la enseñanza de la farmacología en Estados Unidos. Actual Med Peru. 1938; 4(8): 192-8.

14. Gutiérrez-Noriega C. Posición actual de la farmacología en la medicina y en la biología. Actual Med Peru. 1944; 10: $145-52$.

15. Campos HA. Carlos Gutiérrez-Noriega, humanista y pionero de la neurofarmacología y la psiquiatría biológica en el Perú. Rev Peru Med Exp Salud Publica. 2009; 26(4): 577-79.

16. Gutiérrez-Noriega C. Medicina y antropología. Actual Med Peru. 1936; 2(2): 73-4.

17. Gutiérrez-Noriega C. El temperamento cliclotímico y el tipo pícnico entre los aborígenes peruanos. Actual Med Peru. 1936; 1(10): 571-8.

18. Gutiérrez-Noriega C. Biotipología del Perú antiguo. Actual Med Peru. 1936; 2(3): 118-30.

19. Gutiérrez-Noriega C. Diseño de un estudio psicológico y antropológico de la raza Amerindia. Actual Med Peru. 1937; 3(4): 159-95.

20. Gutiérrez-Noriega C. Tres tipos culturales de personalidad. Lima: Imprenta Santa María; 1953.

21. Gutiérrez-Noriega C. Observaciones biotipológicas y psicopatológicas en los enfermos mentales peruanos con referencia a factores raciales y geográficos. Acta Méd Peruana. 1937; 2: 408-31.

22. Gutiérrez-Noriega C. El pensamiento mágico en las pinturas del antiguo Perú. Rev Neuropsiquiatr. 1939; 2(3): 426-61.

23. Gutiérrez-Noriega C. La concepción del mundo y la reacción espiritual congruentes al medio geográfico en el antiguo Perú. En Mariátegui J, Guerra-García R. Cultura y Personalidad - Escritos conexos. Lima: Fondo Editorial Universidad Nacional Mayor de San Marcos; 2010. p. 81-8.

24. Gutiérrez-Noriega C. Sabiduría, humanismo y bondad en Po Chu-i. La Nueva Democracia. 1950; 30(3): 54-60.

25. Mariátegui J, Guerra-García R. Cultura y Personalidad - Escritos conexos. Lima: Fondo Editorial Universidad Nacional Mayor de San Marcos; 2010. p. 81-8.

26. Mariátegui J. La Psiquiatría Peruana en sus figuras Representativas. Lima: Librería Editorial "Minerva"; 1985.

27. Valdivia O. Historia de la Psiquiatría peruana. Lima: Gráfica Morsom; 1964: 217-21.

28. Vallejo A. Literatura y Psiquiatría. Barcelona: Editorial Barna; 1950: 19-66.
29. Gutiérrez-Noriega C. La contribución de Miguel de Cervantes a la psiquiatría. Cuadernos Americanos. 1944; v. $\mathrm{XV} \mathrm{N}^{\circ}$ 3:82-92

30. Gutiérrez-Noriega C. Contribución de Cervantes a la psicología y a la psiquiatría. Rev Neuropsiquiatr. 1944; 7(2): 149-90.

31. Gutiérrez-Noriega C. Cervantes y la Psicología Médica. Rev Neuropsiquiatr. 1946; 9(2): 107-19.

32. Gutiérrez-Noriega C. La personalidad y el carácter en la obra de Cervantes. Rev Neuropsiquiatr. 1947; 10(4): 51641.

33. Gutiérrez-Noriega C. Significado y trascendencia del humorismo en la obra de Cervantes. San Marcos. 1948; 2(4): 43-69.

34. Gutiérrez-Noriega C. Psicologías anormales en la novela picaresca española del siglo de oro. Boletín del Instituto Peruano de Cultura Hispánica. 1948; 3: 163-200.

35. Bailón JM. El cervantismo en dos psiquiatras peruanos: Honorio Delgado y Gutiérrez Noriega. Investigación en Salud. 2003; 5(1): 1-8.

36. Ayala S, Arellano P. Carlos Gutiérrez-Noriega y su contribución a la Escuela Médica Peruana. An Fac med. 2004; 65(2): 147-53.

37. Gutiérrez-Noriega C. Fisiopatología y neurobiología de la epilepsia producida por Cardiazol. [Tesis doctoral]. Lima: Facultad de Medicina. Universidad Nacional Mayor de San Marcos; 1940.

38. Gutiérrez-Noriega C, Zapata OV. Estudios sobre la coca y la cocaína en el Perú. Lima: Ministerio de Educación Pública. Dirección de Educación Artística y Extensión Cultural; 1947: 144 pp.

39. Ayala S, Murillo JP. Gutiérrez-Noriega y el debate en torno al consumo de las hojas de coca (1937-1952). An Fac med. 2018; 79(2): 162-74.

40. Gutiérrez-Noriega C. El cocaísmo y la alimentación en el Perú. An Fac med. 1948; 31(1): 3-92.

41. Castro de la Mata R. Inventario de la coca. Lima: CEDRO; 2003: 280 pp.

42. Bernal E. La obra de Carlos Gutiérrez-Noriega (19061950). Anales de Salud Mental. 2010; 26(1): 75-9.

43. Kozlarek O. Un viraje decisivo. El Correo de la UNESCO. 2011; 64(4): 18-20.

44. Delgado H. Carlos Gutiérrez-Noriega. Necrología. Rev Neuropsiquiatr. 1950; 13(4): 681-85.

43. Murillo JP. Construcción de competencias interculturales para el desarrollo de una propuesta de experiencias de aprendizaje para alumnos de primer año de medicina. An Fac med. 2015; 76: 77-87.

44. Seguin CA. La preparación psicológica del estudiante de medicina. Lima: Universidad Nacional Mayor de San Marcos; 1964. p. 11-57.

45. Nizama M. Humanismo Médico. Revista de la Sociedad Peruana de Medicina Interna [Internet]. 2002 [citado 17 oct 2018]; 15(1). URL disponible en: http://sisbib. unmsm.edu.pe/bvrevistas/spmi/v15n1/humanis_ medico.htm 
46. Zapata V. Nota biográfica. A los 25 años del fallecimiento de Carlos Gutiérrez-Noriega. Acta Méd Peruana. 1975; 4(1-4): 91.

47. Cruz G. La obra de Carlos Gutiérrez-Noriega. Rev Neuropsiquiatr. 1951; 14(1): 134-48.

48. Valdivia O. Panorama de la Psiquiatría en el Perú. Vol 2. Lima: Editorial Universidad Nacional Mayor de San Marcos; 1991: 93-7.
49. Gutiérrez-Noriega C. Discurso de Orden del Sr. Presidente. Rev Neuropsiquiatr. 1946; 9(1): 187-96.

50. Marañón G. Vocación y Ética y Otros Ensayos. 4a ed. Madrid: Espasa-Calpe; 1961: 160 pp.

\section{CORRESPONDENCIA:}

sayalap1@hotmail.com 